\title{
Topological phase transitions in tilted optical lattices
}

\author{
Andrey R. Kolovsky \\ L. V. Kirensky Institute of Physics SB RAS, 660036 Krasnoyarsk, Russia \\ and School of Engineering Physics and Radio Electronics, Siberian Federal University, 660041 Krasnoyarsk, Russia
}

(Received 7 March 2018; published 5 July 2018)

\begin{abstract}
We analyze the energy spectrum and eigenstates of cold atoms in a tilted brick-wall optical lattice. When the tilt is applied, the system exhibits a sequence of topological phase transitions reflected in an abrupt change of the eigenstates. It is demonstrated that these topological phase transitions can be easily detected in a laboratory experiment by observing Bloch oscillations of cold atoms.
\end{abstract}

DOI: 10.1103/PhysRevA.98.013603

\section{INTRODUCTION}

The Bloch band theory is a cornerstone of solid state physics. It gave a simple explanation for the fact that some crystalline materials are electric insulators while the others are conductors. However, after discovery of the quantum Hall effect in 1980 it was gradually recognized that this division of solid crystals into band insulators and metals is incomplete and there are materials which are insulating in the bulk but conducting at the edges. These materials with an unusual Bloch band structure were named topological insulators and their study became a blossoming branch of solid state physics and other fields [1-4].

An excellent playground for studying different models of topological insulators is provided by cold atoms in optical lattices. For example, in Ref. [5] the authors realized the one-dimensional Su-Schrieffer-Heeger (SSH) model [6] and measured the Zak phases for two different dimerization of the SSH lattice. In Ref. [7], the two-dimensional lattice with effective magnetic field was created and the Chern number of the ground magnetic band was measured by observing the anomalous velocity of atoms. The cold atom realization of the Haldane lattice [8] was reported in Ref. [9].

In this work, we discuss a class of topologically nontrivial systems which should be treated as quasi-one-dimensional. These are the tilted bipartite lattices. Topological phase transitions in tilted bipartite lattices were mentioned in Ref. [10], which studied the biased dice lattice. The dice lattice has a rather particular geometry that leads to the Bloch spectrum consisting of three bands, with one band being completely flat. While the emphasis of Ref. [10] was on flat bands, in the present work we focus on the topological phase transitions and their physical manifestations that can be detected in the present-day laboratory experiments with cold atoms. Putting laboratory accessibility on the first place, we consider the brick-wall lattice which was used earlier in the experiment [11] to study the effect of Dirac cones. The network connectivity of the brick-wall lattice is shown in Fig. 1 where the solid and dashed bonds correspond to the strong $J_{1}$ and weak $J_{2}$ coupling, respectively. Here we analyze the simplest case where the brick-wall lattice is tilted in the $y$ direction. In other words, the atoms are subject to a potential field $\mathbf{F}$ aligned with the $y$ axis. The field breaks translational invariance of the system in the $y$ direction and causes the atoms to be localized within the Stark localization length $\sim 1 / F$. However, in the $x$ direction translational symmetry is preserved and, hence, atomic eigenstates are extended Bloch-like states. Localization in one direction together with translational invariance in the other relates the system to the SSH model. However, due to the presence of an extra parameter $F$, the system is essentially richer than the celebrated SSH lattice. In particular, the tilted brick-wall lattice preserves its topological properties even if the hopping matrix element $J_{2}$ is strictly zero.

\section{WANNIER-STARK ENERGY BANDS}

We begin with the energy spectrum of a quantum particle in the tilted brick-wall lattice. As for any bipartite lattice, it consists of pairs of one-dimensional energy bands arranged into the Wannier-Stark ladder [12]; see Fig. 2. Numerically this spectrum can be found by using at least two different methods - by diagonalizing the truncated Hamiltonian matrix or by calculating and diagonalizing the Floquet operator, which is the system evolution operator over the Bloch period. The latter approach has certain advantages over the former because it (i) does not require truncation of the Hilbert space and (ii) opens prospects for analytical studies of the spectrum [12,13].

In the framework of the Floquet operator approach, the eigenstates of a quantum particle in a tilted bipartite lattice are conveniently characterized by the column vector function $\mathbf{Y}(\theta ; \kappa)=\left[Y^{A}(\theta ; \kappa), Y^{B}(\theta ; \kappa)\right]^{T}$ whose Fourier transform gives occupation amplitudes of the lattice sites,

$$
\psi_{l, p}^{A, B} \sim e^{i \kappa d l} \int_{0}^{2 \pi} Y^{A, B}(\theta ; \kappa) e^{i p \theta} d \theta .
$$

In Eq. (1), $d$ denotes the lattice period, $l=x / d$ and $p=y / d$ label the lattice sites, and $\kappa$ is the transverse quasimomentum (i.e., the quasimomentum of the Bloch wave in the $x$ direction). According to Ref. [10], it can be shown that the function $\mathbf{Y}(\theta ; \kappa)$ obeys the ordinary differential equation

$$
i d F \frac{d \mathbf{Y}}{d \theta}=G(\theta ; \kappa) \mathbf{Y}
$$




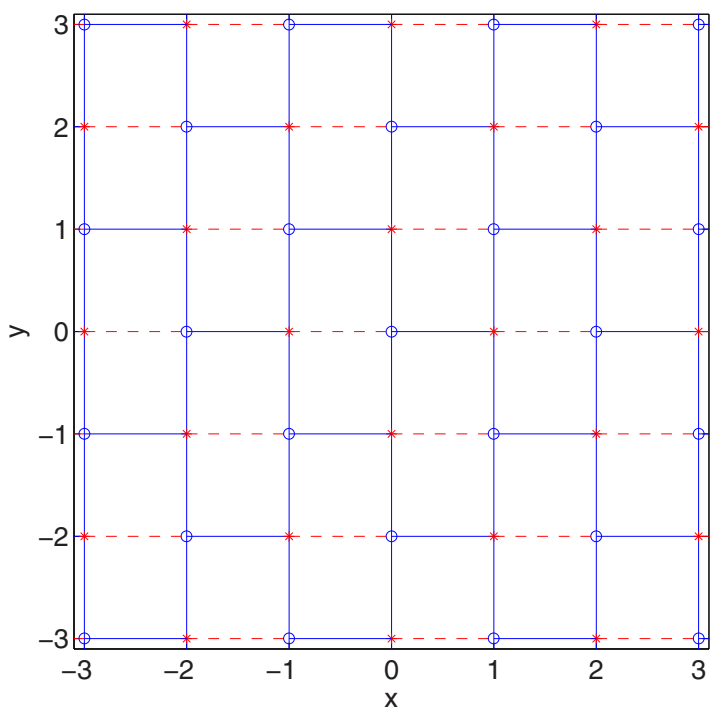

FIG. 1. The brick-wall lattice. The bonds marked by the solid and dashed lines corresponds to the different hoping matrix elements $J_{1}$ and $J_{2}$. Notice that for $J_{2}=0$ the brick-wall lattice transforms into the deformed honeycomb lattice with $A$ and $B$ sites marked by asterisks and open circles.

where the matrix $G(\theta ; \kappa)$ is of the form

$$
G(\theta ; \kappa)=\left(\begin{array}{cc}
0 & f \\
f^{*} & 0
\end{array}\right),
$$

and $f(\theta ; \kappa)=J_{2}+J_{1}\left(e^{i 2 d \kappa}+2 e^{i d \kappa} \cos \theta\right)$. Using Eq. (2), one constructs the $2 \times 2$ unitary (Floquet) matrix

$$
U(\kappa)=\widehat{\exp }\left[\frac{i}{d F} \int_{0}^{2 \pi} G(\theta ; \kappa) d \theta\right]
$$
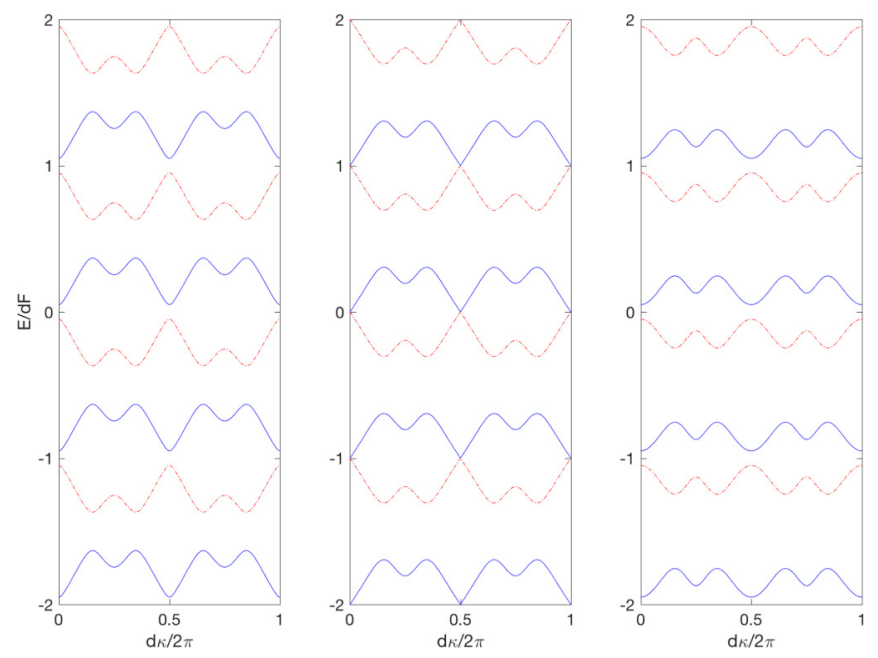

FIG. 2. Energy spectrum of the tilted brick-wall lattice. Parameters are $J_{1}=0.5, J_{2}=0$, and $d F=1 / 1.9$ (left panel), $d F=1 / 2$ (middle panel), and $d F=1 / 2.1$ (right panel). Through the paper, we use arbitrary energy units since only the ratio between the hopping and Stark energies matters.
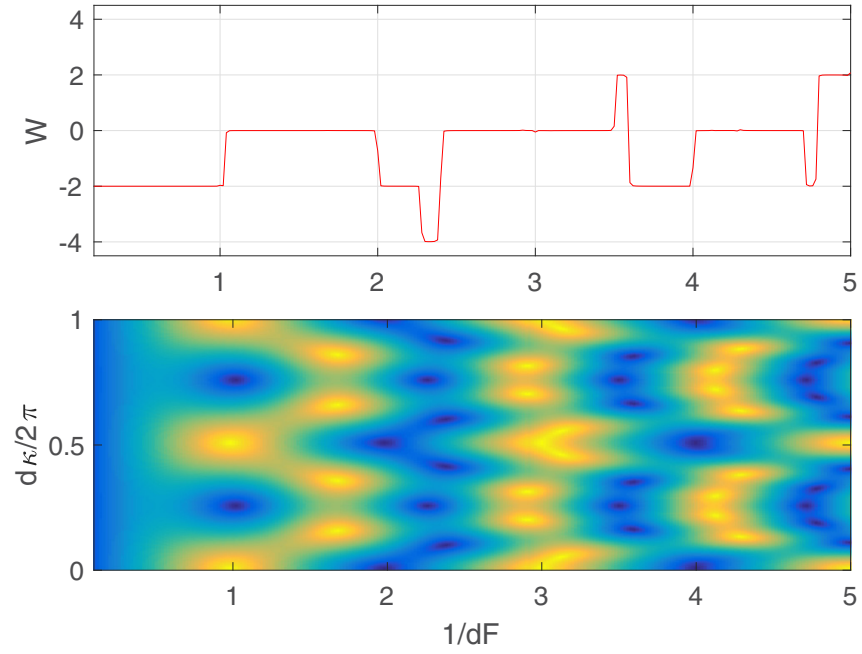

FIG. 3. Upper panel: Winding number of the phase $\chi$ vs the inverse field magnitude. Lower panel: The energy band $E_{n=0}^{(1)}$ as a color map. The limits of the energy axis are $E_{\text {min }}=0$ (dark blue) and $E_{\text {max }}=d F / 2$ (bright yellow). The values of the hopping matrix elements are the same as in Fig. 2.

whose eigenvalues $\lambda_{1}$ and $\lambda_{2}=\lambda_{1}^{*}$ determine the WannierStark spectrum through the relation

$$
E_{n}^{(1,2)}(\kappa)=d F n+i \frac{d F}{2 \pi} \ln \lambda_{1,2}(\kappa)
$$

In what follows, we take the convention that index 1 always refers to the upper band (solid blue lines) and index 2 refers to the lower band (dashed red lines).

We mention in passing the analogy between the WannierStark spectrum of the brick-wall lattice and the Bloch spectrum of the SSH lattice. The latter is known to be given by eigenvalues of the $2 \times 2$ Hermitian matrix

$$
H(\kappa)=\left(\begin{array}{cc}
0 & f \\
f^{*} & 0
\end{array}\right),
$$

where $f(\kappa)=J_{2}+J_{1} e^{i d \kappa}$ [1]. If ratio $J_{2} / J_{1}$ is varied, the Bloch bands develop a conical intersection when $J_{1}=J_{2}$ and the SSH model shows a topological phase transition reflected in a change of the Zak phase. Notice that the existence of this topological invariant is ensured by the particular structure of $H(\kappa)$, which is the same as for the matrix $G(\theta, \kappa)$ in the definition the Floquet operator (4). Thus we may expect a similar phenomenon in the tilted brick-wall lattice where the Wannier-Stark energy bands are sensitive not only to the ratio $J_{1} / J_{2}$ but also to variation of $F$. For future reference, the lower panel in Fig. 3 shows the upper band with the ladder index $n=0$ as a function of the quasimomentum and inverse field magnitude. Our particular interest in this figure are points of conical intersections between energy bands of different symmetry, seen as the dark and bright spots.

\section{TOPOLOGICAL INVARIANT}

We proceed with topological phase transitions. The object to study are eigenvectors $\mathbf{Y}_{1,2}(\kappa)=\left[Y_{1,2}^{A}(\kappa), Y_{1,2}^{B}(\kappa)\right]^{T}$ of the unitary matrix (4). Because of the particular algebraic structure 
of the matrix (3), the eigenvectors can be shown to have the following simple form:

$$
\mathbf{Y}_{1,2}=\left(\begin{array}{c}
\frac{1}{\sqrt{2}} e^{i \chi} \\
\pm \frac{1}{\sqrt{2}} e^{-i \chi}
\end{array}\right)
$$

where $\chi$ is a function of the quasimomentum and without any loss of generality we can require $\chi(\kappa=0)=0$. Following Ref. [10] we introduce a topological invariant-the winding number $W[14]$,

$$
W=\frac{1}{2 \pi i} \int_{0}^{2 \pi} \operatorname{angle}\left(\frac{Y_{j}^{A}}{Y_{j}^{B}}\right) d \kappa=\frac{1}{\pi i} \int_{0}^{2 \pi} e^{-i \chi} \frac{d}{d \kappa} e^{i \chi} d \kappa,
$$

which is an integer number because $\mathbf{Y}_{1,2}(\kappa)$ are periodic functions of the quasimomentum. Notice that $W$ is not affected by gauge transformations when the eigenvectors are multiplied by a phase factor $\exp [i \Phi(\kappa)]$ with $\Phi(\kappa)$ being an arbitrary periodic function of $\kappa$. As $F$ is varied, the winding number (8) may change its value only at some critical values of the field, where energy bands of different symmetry touch each other; see upper panel in Fig. 3. The observed jumps of the winding number is a formal manifestation of topological phase transitions.

Let us focus on the single topological transition, say at $F_{c r}=$ $1 / 2$, where the bands shown in Fig. 2 touch each other at $\kappa=0$ and $\kappa=\pi$. In vicinity of this critical value, the matrix (4) is close to the identity matrix,

$$
U(\kappa=0)=\left(\begin{array}{cc}
\sqrt{1-\epsilon^{2}} & i \epsilon \\
i \epsilon & \sqrt{1-\epsilon^{2}}
\end{array}\right),
$$

where $\epsilon$ is proportional to $\Delta F=F-F_{c r}$. It is easy to see that for $\epsilon \ll 1$ eigenvectors of the matrix (9) are given by Eq. (7) with $\chi=0$ and eigenvalues by $\lambda_{1,2} \approx \exp ( \pm i \epsilon)$. The crucial point is that off-diagonal elements of the matrix (9) change their sign when $F$ crosses $F_{c r}$. Then the symmetric eigenvector $\mathbf{Y}_{1}=[1 / \sqrt{2}, 1 / \sqrt{2}]^{T}$, which has been associated with the upper band, skips to the lower band [16]. This seemingly simple result has important consequences, which can be detected experimentally by inducing Bloch oscillations of atoms in the $x$ direction. We discuss the general scheme of the proposed experiment in the next section.

\section{DETECTING PHASE TRANSITIONS}

We consider a BEC of noninteracting cold atoms (i.e., a coherent wave packet with vanishing phase difference between different sites) and apply an external field $\mathbf{F}$ which is slightly mismatched with the $y$ axis, $F_{x} \ll F_{y} \approx F$. In this setup, the strong component $F_{y}$ creates the ladder of Wannier-Stark energy bands and the weak component $F_{x}$ induces Bloch oscillations of atoms in these bands. These oscillations can be conveniently described in terms of the Wannier-Stark states $\left|\Psi_{n}^{(1,2)}(\kappa)\right\rangle$ as soon as we specify the expansion coefficient $a_{n}^{(1,2)}(\kappa)=\left\langle\Psi_{n}^{(1,2)}(\kappa) \mid \psi(t=0)\right\rangle$. Since the occupation amplitudes of $A$ and $B$ sites in the initial state $|\psi(t=0)\rangle$ have equal phases, we predominantly populate $\kappa=0$ vicinity of upper bands if $F_{y}<F_{c r}$, and $\kappa=0$ vicinity of lower bands
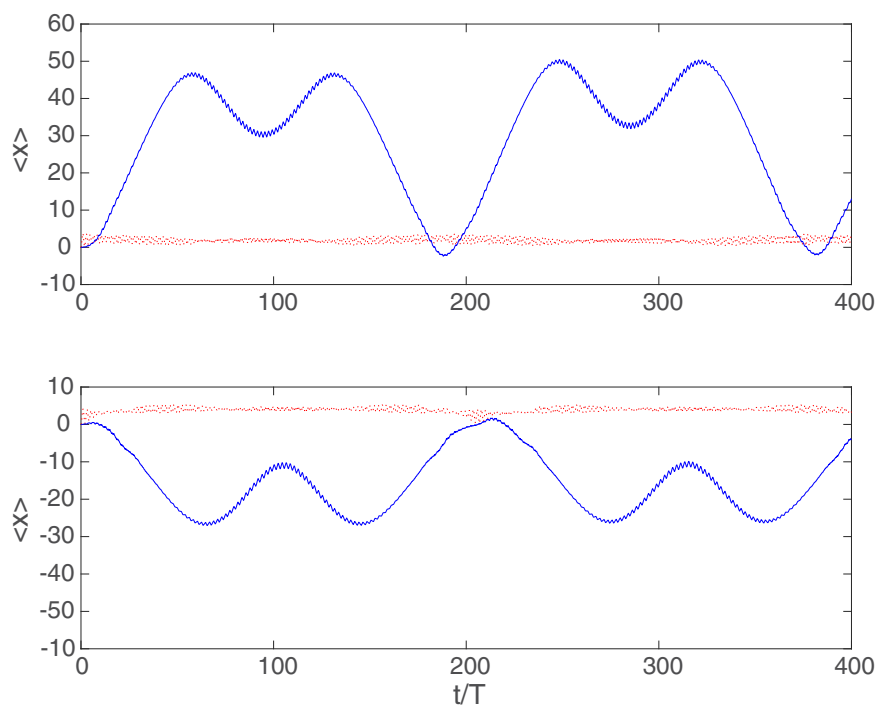

FIG. 4. Bloch oscillations of a localized wave packet for $F=$ $1 / 1.9$ (upper panel) and $F=1 / 2.1$ (lower panel). Time is measured in units of the tunneling period $T=h / J_{1}$. Shown are the mean value $x(t)=\langle\psi(t)|\hat{x}| \psi(t)\rangle$, blue solid lines, and $y(t)=$ $\langle\psi(t)|\hat{y}| \psi(t)\rangle$, red dotted lines. The ratio of the field components $F_{x} / F_{y}=0.005$.

if $F_{y}>F_{c r}$. Thus, the weak component $F_{x}$ induces Bloch oscillations either in upper or lower bands depending on inequality relation between $F$ and $F_{c r}$. This is illustrated in Fig. 4, which shows the results of straightforward numerical simulations of the wave-packet dynamics for $F_{x} / F_{y}=0.005$ and $F=1 / 1.9$, upper panel, and $F=1 / 2.1$, lower panel. It is seen that the wave-packet displacement in the $x$ direction reproduces the dispersion relation of upper bands (blue solid lines) in Fig. 2(a) and lower bands (red dashed lines) in Fig. 2(c), respectively. (Since $d F_{y}>J_{1}$, the wave-packet displacement in the $y$ direction can be neglected.) In our simulations, we intentionally use very small ratio $F_{x} / F_{y}$ since for a larger ratio the Bloch oscillations become complicated by interband Landau-Zenner transitions. Yet, qualitatively the effect remains the same- the wave packet moves in opposite directions for $F$ smaller and larger than $F_{c r}$.

\section{CONCLUSION}

In summary, we analyzed topological phase transitions in the tilted brick-wall optical lattice which are induced by variation of the field magnitude. These transitions correspond to a qualitative change of the two-dimensional WannierStark states and can be detected by observing atomic Bloch oscillations, which nowadays is a routine procedure in the cold-atom physics. We notice, in passing, that one gets similar results for the more familiar honeycomb lattice. Moreover, since in our numerical illustrations we used $J_{2}=0$, the results depicted in Fig. 3 can be directly applied to the honeycomb lattice after rescaling the abscissa axis by the factor $\sqrt{3} / 2$.

To conclude the work, we mention that the discussed phase transitions can be also observed in the curved two-dimensional 
photonic crystals, where the inverse curvature radius plays the role of the field magnitude [17-19]. The latter system also admits to study the other manifestation of topological phase transitions, namely, a rearrangement of the edge states. The detailed analysis of this rearrangement will be given in a separate paper.

\section{ACKNOWLEDGMENTS}

The authors acknowledge fruitful discussions with S. Flach and D. N. Maksimov and the hospitality of Max-Planck Institute for Complex Systems in Dresden where this work was completed.
[1] J. K. Asbóth, L. Oroszlány, and A. Pályi, A Short Course on Topological Insulators: Band Structure and Edge States in One and Two Dimensions, Lecture Notes in Physics Vol. 919 (Springer, Berlin, 2016).

[2] B. A. Bernevig, Topological Insulators and Topological Superconductors (Princeton University Press, Princeton, NJ, 2013).

[3] M. Franz and L. Molenkamp, Topological Insulators, Contemporary Concepts of Condensed Matter Sciences Vol. 6 (Elsevier, Amsterdam, 2013).

[4] S.-Q. Shen, Topological Insulators: Dirac Equation in Condensed Matter, Springer Series in Solid-State Sciences Vol. 187 (Springer, Singapore, 2017).

[5] M. Atala, M. Aidelsburger, J. T. Barreiro, D. Abanin, T. Kitagawa, E. Demler, and I. Bloch, Direct measurement of the Zak phase in topological bloch bands, Nat. Phys. 9, 795 (2013).

[6] W. P. Su, J. R. Schrieffer, and A. J. Heeger, Solitons in Polyacetylene, Phys. Rev. Lett. 42, 1698 (1979).

[7] M. Aidelsburger, M. Lohse, C. Schweizer, M. Atala, J. T. Barreiro, S. Nascimbène, N. R. Cooper, I. Bloch, and N. Goldman, Measuring the Chern number of Hofstadter bands with ultracold bosonic atoms, Nat. Phys. 11, 162 (2015).

[8] F. D. M. Haldane, Model for a Quantum Hall Effect Without Landau Levels: Condensed-Matter Realization of the "Parity Anomaly," Phys. Rev. Lett. 61, 2015 (1988).

[9] G. Jotzu, M. Messer, R. Desbuquois, M. Lebrat, T. Uehlinger, D. Greif, and T. Esslinger, Experimental realization of the topological Haldane model with ultra cold fermions, Nature (London) 515, 237 (2014).

[10] A. R. Kolovsky, A. Ramachandran, and S. Flach, Topological flat Wannier-Stark bands, Phys. Rev. B 97, 045120 (2018).
[11] L. Tarruell, D. Greif, T. Uehlinger, G. Jotzu, and T. Esslinger, Creating, moving, and merging Dirac points with a Fermi gas in a tunable honeycomb lattice, Nature (London) 483, 302 (2012).

[12] D. N. Maksimov, E. N. Bulgakov, and A. R. Kolovsky, WannierStark states in double-periodic lattices. II. Two-dimensional lattices, Phys. Rev. A 91, 053632 (2015).

[13] D. N. Maksimov, E. N. Bulgakov, and A. R. Kolovsky, WannierStark states in double-periodic lattices. I. One-dimensional lattices, Phys. Rev. A 91, 053631 (2015).

[14] Introduced winding number should not be mixed with that discussed in Ref. [15]. The former is a characteristic of the eigenstates of tilted bipartite lattices while the latter is a characteristic of the spectrum of tilted topological lattices (for example, the Haldane lattice) in the limit $F \rightarrow 0$.

[15] W.-R. Lee and K. Park, Direct manifestation of topological order in the winding number of the Wannier-Stark ladder, Phys. Rev. B 92, 195144 (2015).

[16] We mention that this result, when taken together with the fact that eigenvectors are periodic functions of the quasimomentum, necessarily implies a change of the winding number. The latter happens through developing of a nonanalyticity of $\chi=\chi(\kappa)$ at $\kappa=0$ and $\kappa=\pi$.

[17] H. Trompeter, W. Krolikowski, D. N. Neshev, A. S. Desyatnikov, A. A. Sukhorukov, Y. S. Kivshar, T. Pertsch, U. Peschel, and F. Lederer, Bloch oscillations and Zener Tunneling in TwoDimensional Photonic Lattices, Phys. Rev. Lett. 96, 053903 (2006).

[18] A. Szameit, T. Pertsch, S. Nolte, A. Tünnermann, U. Peschel, and F. Lederer, Optical Bloch oscillations in general waveguide lattices, J. Opt. Soc. Am. B 24, 2632 (2007).

[19] G. Corrielli, A. Crespi, G. Della Valle, S. Longhi, and R. Osellame, Fractional Bloch oscillations in photonic lattices, Nat. Commun. 4, 1555 (2013). 